\title{
Recognizing Simple-Triangle Graphs by Restricted 2-Chain Subgraph Cover
}

\author{
Asahi Takaoka \\ Department of Information Systems Creation, Kanagawa University, \\ Rokkakubashi 3-27-1 Kanagawa-ku, Kanagawa, 221-8686, Japan \\ takaoka@jindai.jp
}

\begin{abstract}
A simple-triangle graph (also known as a PI graph) is the intersection graph of a family of triangles defined by a point on a horizontal line and an interval on another horizontal line. The recognition problem for simple-triangle graphs was a longstanding open problem, and recently a polynomial-time algorithm has been given [G. B. Mertzios, The Recognition of Simple-Triangle Graphs and of Linear-Interval Orders is Polynomial, SIAM J. Discrete Math., 29(3):1150-1185, 2015]. Along with the approach of this paper, we show a simpler recognition algorithm for simple-triangle graphs. To do this, we provide a polynomial-time algorithm to solve the following problem: Given a bipartite graph $G$ and a set $F$ of edges of $G$, find a 2-chain subgraph cover of $G$ such that one of two chain subgraphs has no edges in $F$.
\end{abstract}

Keywords: Chain cover, Graph sandwich problem, PI graphs, Simple-triangle graphs, Threshold dimension 2 graphs

\section{Introduction}

Let $L_{1}$ and $L_{2}$ be two horizontal lines in the plane with $L_{1}$ above $L_{2}$. A simple-triangle graph is the intersection graph of a family of triangles spanned by a point on $L_{1}$ and an interval on $L_{2}$. That is, a simple undirected graph is called a simple-triangle graph if there is such a triangle for each vertex and two vertices are adjacent if and only if the corresponding triangles have a nonempty intersection. See Figure 11(a) and 11(b) for example. Simple-triangle graphs are also known as PI graphs [35], where PI stands for Point-Interval. Simple-triangle graphs were introduced in [5] as a generalization of both interval graphs and permutation graphs. Simple-triangle graphs are also known as a proper subclass of trapezoid graphs [5]6], another generalization of interval graphs and permutation graphs.

Recently, the graph isomorphism problem for trapezoid graphs has shown to be isomorphism-complete [23] (that is, polynomial-time equivalent to the problem for general graphs). Since the problem can be solved in linear time for interval graphs [13] and for permutation graphs [4], it has become an interesting question to give the structural characterization of graph classes lying strictly between permutation graphs and trapezoid graphs or between interval graphs and trapezoid graphs [25]. Although a lot of research has been done for interval graphs, for permutation graphs, and for trapezoid 


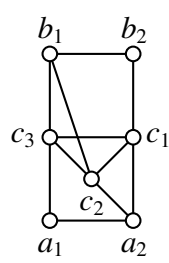

(a) A graph $G$.

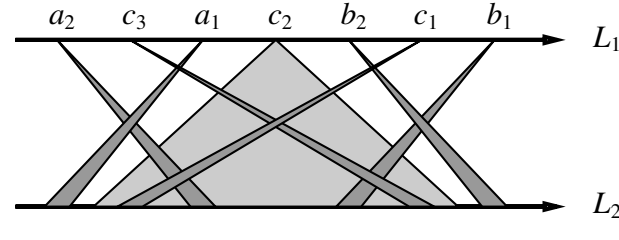

(b) A representation of $G$.

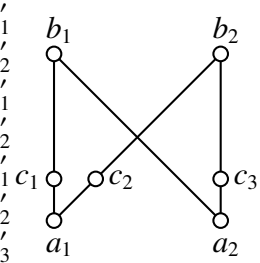

(c) The order $P$.

Fig. 1: A simple-triangle graph $G$, an intersection representation of $G$, and the Hasse diagram of the linear-interval order $P$ obtained from $G$.

graphs (see [22] for example), there are few results for simple-triangle graphs [2|3|5]. It is only recently that a polynomial-time recognition algorithm have been given [17|18].

The recognition algorithm first reduces the recognition problem to the linear-interval cover problem. The algorithm then reduces the linear-interval cover problem to gradually mixed formulas, a tractable subclass of 3-satisfiability (3SAT). Finally, the algorithm solves the gradually mixed formulas by reducing it to 2-satisfiability (2SAT), which can be solved in linear time (see [1] for example). The total running time of the algorithm is $O\left(n^{2} \bar{m}\right)$, where $n$ and $\bar{m}$ are the number of vertices and non-edges of the given graph, respectively.

In this paper, we introduce the restricted 2-chain subgraph cover problem as a generalization of the linear-interval cover problem. Then, we show that our problem is directly reducible to $2 \mathrm{SAT}$. This result does not improve the running time, but it can simplify the previous algorithm for the recognition of simple-triangle graphs.

\subsection{Linear-Interval Cover}

In this section, we briefly describe the linear-interval cover problem and the reduction to it from the recognition problem for simple-triangle graphs. See [18] for the details. We first show that the recognition of simple-triangle graphs is reducible to that of linearinterval orders in $O\left(n^{2}\right)$ time, where $n$ is the number of vertices of the given graph. A partial order is a pair $P=(V, \prec)$, where $V$ is a finite set and $\prec$ is a binary relation on $V$ that is irreflexive and transitive. Partial orders are represented by transitively oriented graphs, which are directed graphs such that if $u \rightarrow v$ and $v \rightarrow w$, then $u \rightarrow w$ for any three vertices $u, v, w$ of the graphs.

There is a correspondence between partial orders and the intersection graphs of geometric objects spanned between two horizontal lines $L_{1}$ and $L_{2}$ [9]. A partial order $P=(V, \prec)$ is called a linear-interval order [2]3] if for each element $v \in V$, there is a triangle $T_{v}$ spanned by a point on $L_{1}$ and an interval on $L_{2}$ such that $u<v$ if and only if $T_{u}$ lies completely to the left of $T_{v}$ for any two elements $u, v \in V$. See Figure [1](b) and 11[c) for example.

For a graph $G=(V, E)$, the graph $\bar{G}=(V, \bar{E})$ is called the complement of $G$, where $u v \in \bar{E}$ if and only if $u v \notin E$ for any pair of vertices $u, v \in V$. We can obtain a linearinterval order from a simple-triangle graph $G$ by giving a transitive orientation to the 
complement $\bar{G}$ of $G$. The complement $\bar{G}$ might have some different transitive orientations, but the following theorem states that any transitive orientation of $\bar{G}$ gives a linear-interval order if $G$ is a simple-triangle graph. A property of partial orders is said to be a comparability invariant if either all orders obtained from the same graph have that property or none have that property.

Theorem 1 ([3]). Being a linear-interval order is a comparability invariant.

Many algorithms have been proposed for transitive orientation, including a linear-time one [16]. Since the complement of a graph can be obtained in $O\left(n^{2}\right)$ time, the recognition of simple-triangle graphs is reducible to that of linear-interval orders in $O\left(n^{2}\right)$ time.

We then show that the recognition of linear-interval orders is reducible to the linearinterval cover problem in $O\left(n^{2}\right)$ time, where $n$ is the number of elements of the given partial orders. Let $P=(V,<)$ be a partial order with $V=\left\{v_{1}, v_{2}, \ldots, v_{n}\right\}$, and let $V^{\prime}=$ $\left\{v_{1}^{\prime}, v_{2}^{\prime}, \ldots, v_{n}^{\prime}\right\}$. The domination bipartite graph $C(P)=\left(V, V^{\prime}, E\right)$ of $P$ is defined such that $v_{i} v_{j}^{\prime} \in E$ if and only if $v_{i}<v_{j}$ in $P$ [14]. We also define that $E_{0}=\left\{v_{i} v_{i}^{\prime} \mid v_{i} \in V\right\}$.

The bipartite complement of $C(P)$ is the bipartite graph $\widehat{C(P)}=\left(V, V^{\prime}, \hat{E}\right)$, where $\hat{E}$ is the set of non-edges between the vertices of $V$ and $V^{\prime}$, that is, $v_{i} v_{j}^{\prime} \in \hat{E}$ if and only if $v_{i} v_{j}^{\prime} \notin E$ for any vertices $v_{i} \in V$ and $v_{j}^{\prime} \in V^{\prime}$. By definition, we have $E_{0} \subseteq \hat{E}$.

Let $2 K_{2}$ denote a graph consisting of four vertices $u_{1}, u_{2}, v_{1}, v_{2}$ with two edges $u_{1} v_{1}, u_{2} v_{2}$. A bipartite graph $G=(U, V, E)$ is called a chain graph [26] if it has no $2 K_{2}$ as an induced subgraph. Equivalently, a bipartite graph $G$ is a chain graph if and only if there is a linear ordering $u_{1}, u_{2}, \ldots, u_{n}$ on $U$ (or $\left.V\right)$ such that $N_{G}\left(u_{1}\right) \subseteq N_{G}\left(u_{2}\right) \subseteq$ $\ldots \subseteq N_{G}\left(u_{n}\right)$, where $N_{G}(u)$ is the set of vertices adjacent to $u$ in $G$. A chain subgraph of $G$ is a subgraph of $G$ that has no induced $2 K_{2}$. A bipartite graph $G=(U, V, E)$ is said to be covered by two chain subgraphs $G_{1}=\left(U, V, E_{1}\right)$ and $G_{2}=\left(U, V, E_{2}\right)$ if $E=E_{1} \cup E_{2}$ (we note that in general, $E_{1}$ and $E_{2}$ are not disjoint), and the pair of chain subgraphs $\left(G_{1}, G_{2}\right)$ is called a 2-chain subgraph cover of $G$. For a partial order $P$, a 2-chain subgraph cover $\left(G_{1}, G_{2}\right)$ of $\widehat{C(P)}$ is called a linear-interval cover if $G_{1}$ has no edges in $E_{0}$.

Theorem 2 ([18]). A partial order $P$ is linear-interval order if and only if $\widehat{C(P)}$ has a linear-interval cover.

The linear-interval cover problem asks whether $\widehat{C(P)}$ has a linear-interval cover. Since $C(P)$ and $\overline{C(P)}$ can be obtained in $O\left(n^{2}\right)$ time from a partial order $P$, the recognition of linear-interval orders is reducible to the linear-interval cover problem in $O\left(n^{2}\right)$ time.

\subsection{Restricted 2-Chain Subgraph Cover}

As a generalization of the linear-interval cover problem, we consider the following restricted problem for 2-chain subgraph cover.

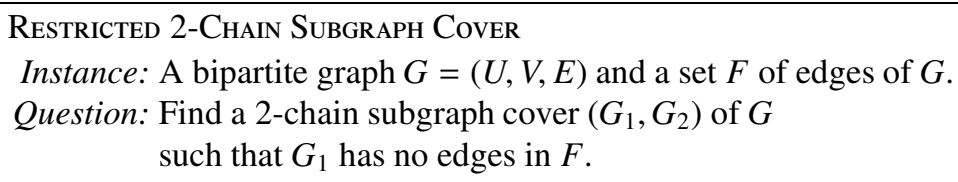




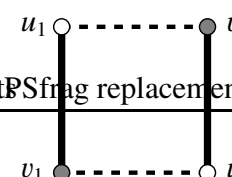

$\left(A_{1}\right)$

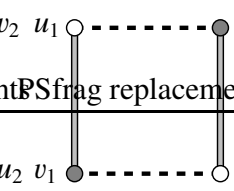

$\left(A_{2}\right)$

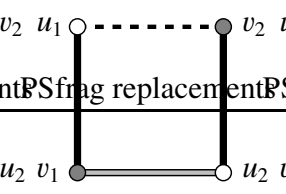

$\left(B_{1}\right)$

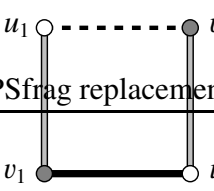

$\left(B_{2}\right)$

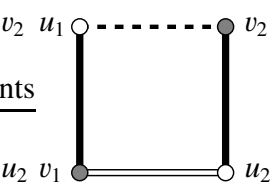

$(C)$

Fig. 2: Forbidden configurations. Solid lines and gray solid lines denote edges in $E_{r}$ and $E_{b}$, respectively. Dashed lines denote non-edges in $\hat{E}$, and double lines denote edges in $F$.

Notice that $G_{2}$ has all the edges in $F$. Let $\hat{E}$ be the set of edges of the bipartite complement $\hat{G}$ of $G$. Let $m=|E|, \hat{m}=|\hat{E}|$, and $f=|F|$. The following is our main result.

Theorem 3. The restricted 2-chain subgraph cover problem can be solved in $O(m \hat{m}+$ $\left.\min \left\{m^{2}, \hat{m}(\hat{m}+f)\right\}\right)$ time.

In the rest of this section, we describe the outline of our algorithm. The details are shown in Section 2 Two edges $e$ and $e^{\prime}$ of a bipartite graph $G=(U, V, E)$ is said to be in conflict in $G$ if the vertices of $e$ and $e^{\prime}$ induce a $2 K_{2}$ in $G$. An edge $e \in E$ is said to be committed if there is another edge $e^{\prime} \in E$ such that $e$ and $e^{\prime}$ are in conflict in $G$, and said to be uncommitted otherwise. Let $E_{c}$ be the set of committed edges of $G$, and let $E_{u}$ be the set of uncommitted edges of $G$.

Suppose $G$ has a 2-chain subgraph $\operatorname{cover}\left(G_{1}, G_{2}\right)$ such that $G_{1}$ has no edges in $F$. If two edges $e, e^{\prime} \in E$ are in conflict in $G$, then $e$ and $e^{\prime}$ may not belong to the same chain subgraph. Therefore, each committed edge in $E_{c}$ belongs to either $G_{1}$ or $G_{2}$. We refer to the committed edges of $G_{1}$ as red edges and the committed edges of $G_{2}$ as blue edges. Let $E_{r}$ and $E_{b}$ be the set of red edges and blue edges, respectively, and we call $\left(E_{r}, E_{b}\right)$ the bipartition of $E_{c}$. Notice that $F \subseteq E_{b} \cup E_{u}$ since $E_{r}$ has no edges in $F$. Hence, we assume without explicitly stating it in the rest of this paper that all the committed edges in $F$ are in $E_{b}$. We can also see that the bipartition $\left(E_{r}, E_{b}\right)$ does not have the following forbidden configurations (see Figure 2).

- Configuration $\left(A_{1}\right)$ [resp., $\left.\left(A_{2}\right)\right]$ consists of four vertices $u_{1}, u_{2} \in U$ and $v_{1}, v_{2} \in V$ with edges $u_{1} v_{1}, u_{2} v_{2} \in E_{r}$ [resp., $u_{1} v_{1}, u_{2} v_{2} \in E_{b}$ ] and non-edges $u_{1} v_{2}, u_{2} v_{1} \in \hat{E}$, that is, $u_{1} v_{1}$ and $u_{2} v_{2}$ are in conflict in $G$;

- Configuration $\left(B_{1}\right)$ [resp., $\left.\left(B_{2}\right)\right]$ consists of four vertices $u_{1}, u_{2} \in U$ and $v_{1}, v_{2} \in V$ with edges $u_{1} v_{1}, u_{2} v_{2} \in E_{r}$ [resp., $u_{1} v_{1}, u_{2} v_{2} \in E_{b}$ ], a non-edge $u_{1} v_{2} \in \hat{E}$, and an edge $u_{2} v_{1} \in E_{b}$ [resp., $u_{2} v_{1} \in E_{r}$ ];

- Configuration $(C)$ consists of four vertices $u_{1}, u_{2} \in U$ and $v_{1}, v_{2} \in V$ with edges $u_{1} v_{1}, u_{2} v_{2} \in E_{r}$, a non-edge $u_{1} v_{2} \in \hat{E}$, and an edge $u_{2} v_{1} \in F$.

Our algorithm construct a bipartition $\left(E_{r}, E_{b}\right)$ of $E_{c}$ that does not have some forbidden configurations. A bipartition of $E_{C}$ is called $(A, C)$-free if it has neither configuration $\left(A_{1}\right),\left(A_{2}\right)$, nor $(C)$. A bipartition of $E_{c}$ is called $(A, B, C)$-free if it has neither configuration $\left(A_{1}\right),\left(A_{2}\right),\left(B_{1}\right),\left(B_{2}\right)$, nor $(C)$. 
Theorem 4. A bipartite graph $G$ has a 2-chain subgraph cover $\left(G_{1}, G_{2}\right)$ such that $G_{1}$ has no edges in $F$ if and only if $E_{c}$ has an $(A, C)$-free bipartition.

The outline of our algorithm is as follows.

Step 1: Partition the set $E_{c}$ of committed edges into an $(A, C)$-free bipartition $\left(E_{r}, E_{b}\right)$ by solving $2 \mathrm{SAT}$.

Step 2: From the $(A, C)$-free bipartition $\left(E_{r}, E_{b}\right)$ of $E_{c}$, compute an $(A, B, C)$-free bipartition $\left(E_{r}^{\prime}, E_{b}^{\prime}\right)$ of $E_{c}$ by swapping some edges between $E_{r}$ and $E_{b}$.

Step 3: From the $(A, B, C)$-free bipartition $\left(E_{r}^{\prime}, E_{b}^{\prime}\right)$ of $E_{c}$, compute a desired 2-chain subgraph cover of $G$ by adding some uncommitted edges into $E_{r}^{\prime}$ and $E_{b}^{\prime}$.

We will show in Sections 2.1, 2.2 and 2.3 that Step 1, Step 2, and Step 3 can be done in $O\left(\min \left\{m^{2}, \hat{m}(\hat{m}+f)\right\}\right)$ time, $O(m \hat{m})$ time, and linear time, respectively.

\subsection{Related Work}

A bipartite graph $G=(U, V, E)$ is said to be covered by $k$ subgraphs $G_{i}=\left(U, V, E_{i}\right)$, $1 \leq i \leq k$, if $E=E_{1} \cup E_{2} \cup \cdots \cup E_{k}$. A $k$-chain subgraph cover problem asks whether a given bipartite graph can be covered by $k$ chain subgraphs. The $k$-chain subgraph cover problem is NP-complete if $k \geq 3$, while it is polynomial-time solvable if $k \leq 2$ [26].

The 2-chain subgraph cover problem is closely related to some recognition problems; more precisely, they can be efficiently reduced to the 2-chain subgraph cover problem. They are the recognition problems for threshold dimension 2 graphs on split graphs [11|19], circular-arc graphs with clique cover number 2 [10|21], 2-directional orthogonal ray graphs [20[24], and trapezoid graphs [14]. Other related problems and surveys can be found in Chapter 8 of [15] and Section 13.5 of [22].

As far as we know, there are two approaches for the 2-chain subgraph cover problem and the other related problems. One approach is shown in [14]21], which reduces the 2chain subgraph cover problem to the recognition of 2-dimensional partial orders. This approach is used in the fastest known algorithm [14] with a running time of $O\left(n^{2}\right)$, where $n$ is the number of vertices of the given graph. Another approach can be found in [10]11[19]. They show that a bipartite graph $G=(U, V, E)$ has a 2-chain subgraph cover if and only if the conflict graph $G^{*}=\left(V^{*}, E^{*}\right)$ of $G$ is bipartite, where $V^{*}=E$ and two edges $e$ and $e^{\prime}$ in $E$ are adjacent in $G^{*}$ if $e$ and $e^{\prime}$ are in conflict in $G$. We note that the algorithm in this paper is based on the latter approach.

In Section 8.6 of [15], the following problem is considered for recognizing threshold dimension 2 graphs: Given a bipartite graph $G$ and a pair $\left(F_{1}, F_{2}\right)$ of edge sets, find a 2-chain subgraph cover $\left(G_{1}, G_{2}\right)$ of $G$ such that $G_{1}$ and $G_{2}$ have every edge in $F_{1}$ and $F_{2}$, respectively. We call such a problem the extension problem for 2-chain subgraph cover. We emphasize that the extension problem is not a generalization of our restricted 2-chain subgraph cover problem since in the extension problem, $G_{1}$ and $G_{2}$ are allowed to have all the uncommitted edges of $G$. As shown in [15], this problem can be solved in polynomial time by reducing it to some variation of the recognition problem for 2-dimensional partial orders. We note that this variation can be stated as the problem of extending a partial orientation of a permutation graph to a 2-dimensional partial order [12]. 


\section{Algorithm}

\subsection{Partitioning Edges}

A $2 C N F$ formula is a Boolean formula in conjunctive normal form with at most two literals per clause. In this section, we construct a $2 \mathrm{CNF}$ formula $\phi$ such that $\phi$ is satisfiable if and only if $G$ has an $(A, C)$-free bipartition of $E_{c}$. The construction of $\phi$ is as follows:

- Assign the Boolean variable $x_{e}$ to each committed edge $e \in E_{c}$;

- Add the clause $\left(x_{e}\right)$ for each edge $e \in F \cap E_{c}$;

- For each pair of two edges $e$ and $e^{\prime}$ in $E_{c}$, add the clauses $\left(x_{e} \vee x_{e^{\prime}}\right)$ and $\left(\overline{x_{e}} \vee \overline{x_{e^{\prime}}}\right)$ to $\phi$ if $e$ and $e^{\prime}$ are in conflict in $G$;

- For each pair of two edges $e$ and $e^{\prime}$ in $E_{c}$, add the clause $\left(x_{e} \vee x_{e^{\prime}}\right)$ to $\phi$ if the vertices of $e$ and $e^{\prime}$ induce a path of length 3 whose middle edge is in $F$ (see the forbidden configuration $(C)$ in Figure 2).

Then, we obtain the bipartition $\left(E_{r}, E_{b}\right)$ of $E_{c}$ from a truth assignment $\tau$ of the variables as follows:

$-x_{e}=0$ in $\tau \Longleftrightarrow e \in E_{r}$ (or $x_{e}=1$ in $\left.\tau \Longleftrightarrow e \in E_{b}\right)$.

It is obvious that a truth assignment $\tau$ satisfies $\phi$ if and only if the corresponding bipartition of $E_{c}$ is $(A, C)$-free and all the committed edges in $F$ are in $E_{b}$.

The 2CNF formula $\phi$ has at most $m$ Boolean variables. We can also see that $\phi$ has at most $f+2 \cdot \min \left\{m^{2}, \hat{m}(\hat{m}+f)\right\}$ clauses since $\phi$ has at most two clauses for each pair of two edges in $E_{c}$ or for each pair of a non-edge in $\hat{E}$ and an edge in $F$. Then, $\phi$ can be obtained in $O\left(\min \left\{m^{2}, \hat{m}(\hat{m}+f)\right\}\right)$ time from $G$ and $F$. Since a satisfying truth assignment of a $2 \mathrm{CNF}$ formula can be computed in linear time (see [1] for example), we have the following.

Lemma 1. An $(A, C)$-free bipartition of $E_{c}$ can be computed in $O\left(\min \left\{m^{2}, \hat{m}(\hat{m}+f)\right\}\right)$ time.

\subsection{Swapping Edges}

In this section, we show an $O(m \hat{m})$-time algorithm to transform a given $(A, C)$-free bipartition $\left(E_{r}, E_{b}\right)$ of $E_{c}$ into an $(A, B, C)$-free bipartition $\left(E_{r}^{\prime}, E_{b}^{\prime}\right)$ of $E_{c}$. For a nonedge $u v \in \hat{E}$, we define that

$$
\begin{aligned}
H_{r} & =\left\{u^{\prime} v^{\prime} \in E_{r} \mid u v^{\prime}, u^{\prime} v \in E_{b}\right\} ; \\
H_{b} & =\left\{u^{\prime} v^{\prime} \in E_{b} \mid u v^{\prime}, u^{\prime} v \in E_{r}\right\} ; \\
H & =H_{r} \cup H_{b} .
\end{aligned}
$$

In other words, $H_{r}$ is the set of red edges of all configurations $\left(B_{2}\right)$ having non-edge $u v$, and $H_{b}$ is the set of blue edges of all configurations $\left(B_{1}\right)$ having non-edge $u v$. Between $E_{r}$ and $E_{b}$, we swap all edges in $H$ to obtain another bipartition $\left(E_{r}^{\prime}, E_{b}^{\prime}\right)$ of $E_{c}$, that is, we define that

$$
\begin{aligned}
& E_{r}^{\prime}=\left(E_{r} \backslash H_{r}\right) \cup H_{b} ; \\
& E_{b}^{\prime}=\left(E_{b} \backslash H_{b}\right) \cup H_{r} .
\end{aligned}
$$




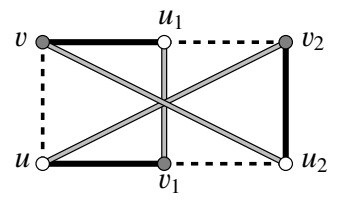

(a) Case 1-1 in Lemma 2

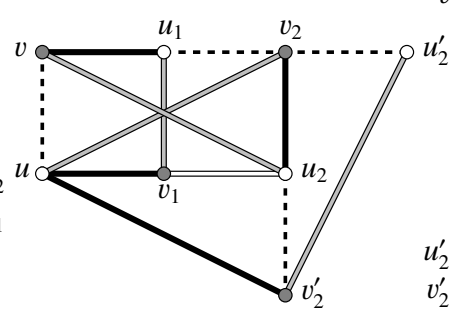

(d) Case 5-1 in Lemma2

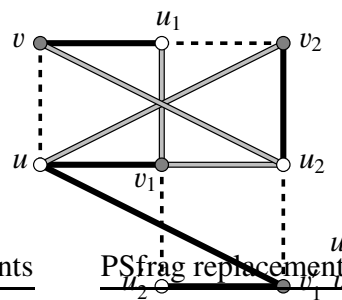

(b) Case 3-1 in Lemma 2

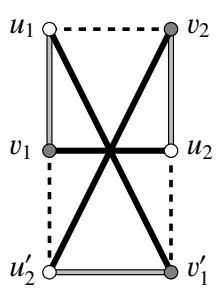

(e) Case 2 in Lemma 4

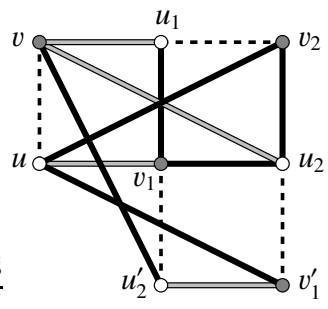

(c) Case 3-3 in Lemma 2

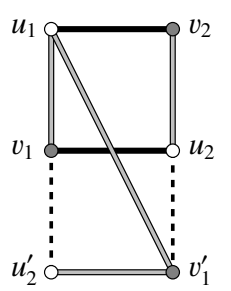

(f) Case 3 in Lemma 4

Fig. 3: Illustrating the proof of cases in Lemmas 2 and 4 Lines denote the same type of edges as in Figure 2

Since $\left(E_{r}, E_{b}\right)$ is $(A, C)$-free, we have $F \cap H=\emptyset$. Hence, all the committed edges in $F$ remain blue in the new bipartition $\left(E_{r}^{\prime}, E_{b}^{\prime}\right)$. Notice that by swapping the edges, we remove all the configurations $\left(B_{1}\right)$ and $\left(B_{2}\right)$ having non-edge $u v \in \hat{E}$. We claim that the swapping generates no forbidden configurations.

Lemma 2. No edges in $H$ is an edge of any forbidden configurations of the new bipartition $\left(E_{r}^{\prime}, E_{b}^{\prime}\right)$ of $E_{c}$.

Proof. We assume that the new bipartition $\left(E_{r}^{\prime}, E_{b}^{\prime}\right)$ has some configuration with at least one edge in $H$, and obtain a contradiction.

Case 1: Suppose $\left(E_{r}^{\prime}, E_{b}^{\prime}\right)$ has a configuration $\left(A_{1}\right)$, that is, there are four vertices $u_{1}, v_{1}, u_{2}, v_{2}$ with $u_{1} v_{1}, u_{2} v_{2} \in E_{r}^{\prime}$ and $u_{1} v_{2}, u_{2} v_{1} \in \hat{E}$.

Case 1-1: Suppose $u_{1} v_{1} \in H$ and $u_{2} v_{2} \notin H$. This implies that $u_{1} v_{1} \in E_{b}$ and $u_{2} v_{2}, u v_{1}, u_{1} v \in E_{r}$. See Figure [3[a), We have $u v_{2} \in E$, for otherwise $u v_{1} \in E_{r}$ and $u_{2} v_{2} \in E_{r}$ would be in conflict in $G$. Since $u v_{2}$ and $u_{1} v \in E_{r}$ are in conflict in $G$, we have $u v_{2} \in E_{b}$. Similarly, we have $u_{2} v \in E$, for otherwise $u_{1} v \in E_{r}$ and $u_{2} v_{2} \in E_{r}$ would be in conflict in $G$. Since $u_{2} v$ and $u v_{1} \in E_{r}$ are in conflict in $G$, we have $u_{2} v \in E_{b}$. However, we have from $u v_{2}, u_{2} v \in E_{b}$ that $u_{2} v_{2} \in H_{r}$, a contradiction.

Case 1-2: Suppose $u_{2} v_{2} \in H$ and $u_{1} v_{1} \notin H$. This case is symmetric to Case 1-1.

Case 1-3: Suppose $u_{1} v_{1}, u_{2} v_{2} \in H$. This implies that $u_{1} v_{1}, u_{2} v_{2} \in E_{b}$ and $u v_{1}, u_{1} v$, $u v_{2}, u_{2} v \in E_{r}$, but $u_{1} v \in E_{r}$ and $u v_{2} \in E_{r}$ are in conflict in $G$, a contradiction.

Case 2: Suppose $\left(E_{r}^{\prime}, E_{b}^{\prime}\right)$ has a configuration $\left(A_{2}\right)$. This case is symmetric to Case 1.

Case 3: Suppose $\left(E_{r}^{\prime}, E_{b}^{\prime}\right)$ has a configuration $\left(B_{1}\right)$, that is, there are four vertices $u_{1}, v_{1}, u_{2}, v_{2}$ with $u_{1} v_{1}, u_{2} v_{2} \in E_{r}^{\prime}, u_{2} v_{1} \in E_{b}^{\prime}$, and $u_{1} v_{2} \in \hat{E}$. 
Case 3-1: Suppose $u_{1} v_{1} \in H$ and $u_{2} v_{2}, u_{2} v_{1} \notin H$. This implies that $u_{1} v_{1}, u_{2} v_{1} \in E_{b}$ and $u_{2} v_{2}, u v_{1}, u_{1} v \in E_{r}$. See Figure [b). We have $u_{2} v \in E$, for otherwise $u_{1} v \in E_{r}$ and $u_{2} v_{2} \in E_{r}$ would be in conflict in $G$. If $u_{2} v \in E_{r}$, then we have from $u v_{1} \in E_{r}$ that $u_{2} v_{1} \in H_{b}$, a contradiction. Therefore, $u_{2} v \in E_{b} \cup E_{u}$. Since $u_{2} v_{1} \in E_{b}$, there is an edge $u_{2}^{\prime} v_{1}^{\prime} \in E_{r}$ such that $u_{2} v_{1}$ and $u_{2}^{\prime} v_{1}^{\prime}$ are in conflict in $G$, that is, $u_{2} v_{1}^{\prime}, u_{2}^{\prime} v_{1} \in \hat{E}$. We have $u v_{1}^{\prime} \in E$, for otherwise $u v_{1} \in E_{r}$ and $u_{2}^{\prime} v_{1}^{\prime} \in E_{r}$ would be in conflict in $G$. Since $u v_{1}^{\prime}$ and $u_{2} v \in E_{b} \cup E_{u}$ are in conflict in $G$, we have $u v_{1}^{\prime} \in E_{r}$ and $u_{2} v \in E_{b}$. Then, we have $u v_{2} \in E$, for otherwise $u v_{1}^{\prime} \in E_{r}$ and $u_{2} v_{2} \in E_{r}$ would be in conflict in $G$. Since $u v_{2}$ and $u_{1} v \in E_{r}$ are in conflict in $G$, we have $u v_{2} \in E_{b}$. However, we have from $u v_{2}, u_{2} v \in E_{b}$ that $u_{2} v_{2} \in H_{r}$, a contradiction.

Case 3-2: Suppose $u_{2} v_{2} \in H$ and $u_{1} v_{1}, u_{2} v_{1} \notin H$. This case is symmetric to Case 3-1.

Case 3-3: Suppose $u_{2} v_{1} \in H$ and $u_{1} v_{1}, u_{2} v_{2} \notin H$. This implies that $u_{1} v_{1}, u_{2} v_{2}, u_{2} v_{1} \in$ $E_{r}$ and $u v_{1}, u_{2} v \in E_{b}$. See Figure 3[(c)] Since $u_{2} v_{1} \in E_{r}$, there is an edge $u_{2}^{\prime} v_{1}^{\prime} \in E_{b}$ such that $u_{2} v_{1}$ and $u_{2}^{\prime} v_{1}^{\prime}$ are in conflict in $G$, that is, $u_{2} v_{1}^{\prime}, u_{2}^{\prime} v_{1} \in \hat{E}$. We have $u v_{1}^{\prime} \in E$, for otherwise $u v_{1} \in E_{b}$ and $u_{2}^{\prime} v_{1}^{\prime} \in E_{b}$ would be in conflict in $G$. Since $u v_{1}^{\prime}$ and $u_{2} v \in E_{b}$ are in conflict in $G$, we have $u v_{1}^{\prime} \in E_{r}$. Then, we have $u v_{2} \in E$, for otherwise $u v_{1}^{\prime} \in E_{r}$ and $u_{2} v_{2} \in E_{r}$ would be in conflict in $G$. If $u v_{2} \in E_{b}$, then we have from $u_{2} v \in E_{b}$ that $u_{2} v_{2} \in H_{r}$, a contradiction. Therefore, $u v_{2} \in E_{r} \cup E_{u}$. Similarly, we have $u_{2}^{\prime} v \in E$, for otherwise $u_{2} v \in E_{b}$ and $u_{2}^{\prime} v_{1}^{\prime} \in E_{b}$ would be in conflict in $G$. Since $u_{2}^{\prime} v$ and $u v_{1} \in E_{b}$ are in conflict in $G$, we have $u_{2}^{\prime} v \in E_{r}$. Then, we have $u_{1} v \in E$, for otherwise $u_{1} v_{1} \in E_{r}$ and $u_{2}^{\prime} v \in E_{r}$ would be in conflict in $G$. Since $u_{1} v$ and $u v_{2} \in E_{r} \cup E_{u}$ are in conflict in $G$, we have $u_{1} v \in E_{b}$ and $u v_{2} \in E_{r}$. However, we have from $u v_{1} \in E_{b}$ that $u_{1} v_{1} \in H_{r}$, a contradiction.

Case 3-4: Suppose $u_{1} v_{1}, u_{2} v_{2} \in H$ and $u_{2} v_{1} \notin H$. We have a contradiction as Case 1-3.

Case 3-5: Suppose $u_{1} v_{1}, u_{2} v_{1} \in H$ and $u_{2} v_{2} \notin H$. This implies that $u_{1} v_{1} \in H_{b}$ and $u_{2} v_{1} \in H_{r}$, but it follows that $u v_{1} \in E_{r}$ from $u_{1} v_{1} \in H_{b}$ and $u v_{1} \in E_{b}$ from $u_{2} v_{1} \in H_{r}$, a contradiction.

Case 3-6: Suppose $u_{2} v_{2}, u_{2} v_{1} \in H$ and $u_{1} v_{1} \notin H$. This case is symmetric to Case 3-5.

Case 3-7: Suppose $u_{1} v_{1}, u_{2} v_{2}, u_{2} v_{1} \in H$. We have a contradiction as Case 3-5.

Case 4: Suppose $\left(E_{r}^{\prime}, E_{b}^{\prime}\right)$ has a configuration $\left(B_{2}\right)$. This case is symmetric to Case 3 .

Case 5: Suppose $\left(E_{r}^{\prime}, E_{b}^{\prime}\right)$ has a configuration $(C)$, that is, there are four vertices $u_{1}, v_{1}, u_{2}, v_{2}$ with $u_{1} v_{1}, u_{2} v_{2} \in E_{r}^{\prime}, u_{1} v_{2} \in \hat{E}$, and $u_{2} v_{1} \in F$. Since the bipartition $\left(E_{r}, E_{b}\right)$ is $(A, C)$-free, we have $u_{2} v_{1} \notin H$.

Case 5-1: Suppose $u_{1} v_{1} \in H$ and $u_{2} v_{2} \notin H$. This implies that $u_{1} v_{1} \in E_{b}$ and $u_{2} v_{2}, u v_{1}, u_{1} v \in E_{r}$. See Figure [3] We have $u v_{2} \in E$, for otherwise the vertices $u, v_{1}, u_{2}, v_{2}$ would induce a configuration $(C)$. Since $u v_{2}$ and $u_{1} v \in E_{r}$ are in conflict in $G$, we have $u v_{2} \in E_{b}$. Similarly, we have $u_{2} v \in E$, for otherwise $u_{1} v \in E_{r}$ and $u_{2} v_{2} \in E_{r}$ would be in conflict in $G$. If $u_{2} v \in E_{r}$, then the vertices $u, v_{1}, u_{2}, v$ would induce a configuration $(C)$. Therefore, $u_{2} v \in E_{b} \cup E_{u}$. Since $u_{2} v_{2} \in E_{r}$, there is an edge $u_{2}^{\prime} v_{2}^{\prime} \in E_{b}$ such that $u_{2} v_{2}$ and $u_{2}^{\prime} v_{2}^{\prime}$ are in conflict in $G$, that is, $u_{2} v_{2}^{\prime}, u_{2}^{\prime} v_{2} \in \hat{E}$. We have $u v_{2}^{\prime} \in E$, for otherwise $u_{2}^{\prime} v_{2}^{\prime} \in E_{b}$ and $u v_{2} \in E_{b}$ would be in conflict in $G$. Since $u v_{2}^{\prime}$ and $u_{2} v \in E_{b} \cup E_{u}$ are in conflict in $G$, we have $u v_{2}^{\prime} \in E_{r}$ and $u_{2} v \in E_{b}$. However, we have from $u v_{2}, u_{2} v \in E_{b}$ that $u_{2} v_{2} \in H_{r}$, a contradiction.

Case 5-2: Suppose $u_{2} v_{2} \in H$ and $u_{1} v_{1} \notin H$. This case is symmetric to Case 5-1. 
Case 5-3: Suppose $u_{1} v_{1}, u_{2} v_{2} \in H$. We have a contradiction as Case 1-3.

Since all the cases above lead to contradictions, we conclude that the new bipartition $\left(E_{r}^{\prime}, E_{b}^{\prime}\right)$ has no forbidden configurations with an edge in $H$.

It follows from Lemma 2 that continuing in this way for each non-edge in $\hat{E}$, we can obtain an $(A, B, C)$-free bipartition of $E_{c}$. Since the set $H$ can be computed in $O(m)$ time for each non-edge in $\hat{E}$, the overall running time is $O(m \hat{m})$.

Lemma 3. From a given $(A, C)$-free bipartition of $E_{c}$, an $(A, B, C)$-free bipartition of $E_{c}$ can be computed in $O(m \hat{m})$ time.

\subsection{Adding edges}

In this section, we claim that a given $(A, B, C)$-free bipartition $\left(E_{r}, E_{b}\right)$ of $E_{c}$ can be extended in linear time into a 2 -chain subgraph cover $\left(G_{1}, G_{2}\right)$ of $G$ such that $G_{1}$ has no edges in $F$. We first show the following.

Lemma 4. The subgraph of $G$ induced by $E_{b} \cup E_{u}$ is a chain graph.

Proof. We show that no $2 K_{2}$ is in the subgraphs of $G$ induced by $E_{b} \cup E_{u}$.

Case 1: Suppose $u_{1} v_{1}, u_{2} v_{2} \in E_{b} \cup E_{u}$ and $u_{1} v_{2}, u_{2} v_{1} \in \hat{E}$. It is obvious that $u_{1} v_{1}, u_{2} v_{2} \notin E_{u}$, but $u_{1} v_{1}, u_{2} v_{2} \in E_{b}$ implies that the vertices $u_{1}, v_{1}, u_{2}, v_{2}$ induce a configuration $\left(A_{2}\right)$, a contradiction.

Case 2: Suppose $u_{1} v_{1}, u_{2} v_{2} \in E_{b} \cup E_{u}, u_{1} v_{2} \in \hat{E}$, and $u_{2} v_{1} \in E \backslash\left(E_{b} \cup E_{u}\right)$. Since $u_{2} v_{1} \in E \backslash\left(E_{b} \cup E_{u}\right)=E_{r}$, there is an edge $u_{2}^{\prime} v_{1}^{\prime} \in E_{b}$ such that $u_{2} v_{1}$ and $u_{2}^{\prime} v_{1}^{\prime}$ are in conflict in $G$, that is, $u_{2}^{\prime} v_{1}, u_{2} v_{1}^{\prime} \in \hat{E}$. See Figure 3 (e). We have $u_{1} v_{1}^{\prime} \in E$, for otherwise the vertices $u_{1}, v_{1}, u_{2}^{\prime}, v_{1}^{\prime}$ would induce a configuration in Case 1 . Since $u_{1} v_{1}^{\prime}$ and $u_{2} v_{2} \in$ $E_{b} \cup E_{u}$ are in conflict in $G$, we have $u_{2} v_{2} \in E_{b}$. Similarly, we have $u_{2}^{\prime} v_{2} \in E$, for otherwise the vertices $u_{2}, v_{2}, u_{2}^{\prime}, v_{1}^{\prime}$ would induce a configuration in Case 1 . Since $u_{2}^{\prime} v_{2}$ and $u_{1} v_{1} \in E_{b} \cup E_{u}$ are in conflict in $G$, we have $u_{1} v_{1} \in E_{b}$, but then the vertices $u_{1}, v_{1}, u_{2}, v_{2}$ induce a configuration $\left(B_{2}\right)$, a contradiction.

Case 3: Suppose $u_{1} v_{1}, u_{2} v_{2} \in E_{b} \cup E_{u}$ and $u_{1} v_{2}, u_{2} v_{1} \in E \backslash\left(E_{b} \cup E_{u}\right)$. Since $u_{2} v_{1} \in$ $E \backslash\left(E_{b} \cup E_{u}\right)=E_{r}$, there is an edge $u_{2}^{\prime} v_{1}^{\prime} \in E_{b}$ such that $u_{2} v_{1}$ and $u_{2}^{\prime} v_{1}^{\prime}$ are in conflict in $G$, that is, $u_{2}^{\prime} v_{1}, u_{2} v_{1}^{\prime} \in \hat{E}$. See Figure $3\left(\right.$ f) We have $u_{1} v_{1}^{\prime} \notin \hat{E} \cup E_{r}$, for otherwise the vertices $u_{1}, v_{1}, u_{2}^{\prime}, v_{1}^{\prime}$ would induce a configuration in Case 1 or Case 2. However, $u_{1} v_{1}^{\prime} \in E_{b} \cup E_{u}$ implies that the vertices $u_{2}, v_{2}, u_{1}, v_{1}^{\prime}$ induce a configuration in Case 2, a contradiction.

Since all the cases above lead to contradictions, we conclude that the subgraph of $G$ induced by $E_{b} \cup E_{u}$ has no $2 K_{2}$, and it is a chain subgraph of $G$.

We next show that $E_{r}$ can be extended into a chain graph in $G-F$, the subgraph of $G$ obtained by removing all the edges in $F$. To do this, we consider the following problem: Given a graph $H$ and a set $M$ of edges of $H$, find a chain subgraph $C$ of $H$ containing all edges in $M$. This problem is called the chain graph sandwich problem, and the chain graph $C$ is called a chain completion of $M$ in $H$. Although the chain graph sandwich problem is NP-complete, it can be solved in linear time if $H$ is a bipartite graph [7]. The chain graph sandwich problem on bipartite graphs is closely related to 
the threshold graph sandwich problem [8]19] (see also Section 1.5 of [15]), and in the proof of Lemma 5, we will use an argument similar to that used in the literature.

Let $H=(U, V, E)$ be a bipartite graph, let $\hat{E}$ be the set of edges of the bipartite complement $\hat{H}$ of $H$, and let $k \geq 2$. A set of $k$ distinct vertices $u_{0}, u_{1}, \ldots, u_{k-1}$ in $U$ and $k$ distinct vertices $v_{0}, v_{1}, \ldots, v_{k-1}$ in $V$ is called an alternating cycle of $M$ relative to $H$ if $u_{i} v_{i} \in \hat{E}$ and $u_{i+1} v_{i} \in M$ for any $i, 0 \leq i<k$ (indices are modulo $k$ ). Note that an alternating cycle of $M$ with lengh 4 relative to $H$ is exactly a $2 K_{2}$ of $M$ in $H$.

Lemma 5. Let $M$ be a set of edges in a bipartite graph $H$.

- The set $M$ of edges has a chain completion in $H$ if and only if there are no alternating cycles of $M$ relative to $H$.

- The chain completion of $M$ in $H$ can be computed in $O(n+m)$ time.

Proof. The proof is in Appendix. The details of the algorithm are also shown in [7].

Then, we show that $E_{r}$ has a chain completion in $G-F$.

Lemma 6. There are no alternating cycles of $E_{r}$ relative to $G-F$.

Proof. We first prove that there are no alternating cycles of $E_{r}$ with length 4 relative to $G-F$, that is, no two edges in $E_{r}$ are in conflict in $G-F$. Since the bipartition $\left(E_{r}, E_{b}\right)$ does not have a configuration $\left(A_{1}\right)$ or $(C)$, it is enough to show that $\left(E_{r}, E_{b}\right)$ has no configuration consisting of four vertices $u_{1}, v_{1}, u_{2}, v_{2}$ with edges $u_{1} v_{1}, u_{2} v_{2} \in E_{r}$ and $u_{1} v_{2}, u_{2} v_{1} \in F$. Suppose $\left(E_{r}, E_{b}\right)$ has such a configuration. Since $u_{1} v_{1} \in E_{r}$, there is an edge $u_{1}^{\prime} v_{1}^{\prime} \in E_{b}$ such that $u_{1} v_{1}$ and $u_{1}^{\prime} v_{1}^{\prime}$ are in conflict in $G$, that is, $u_{1} v_{1}^{\prime}, u_{1}^{\prime} v_{1} \in \hat{E}$. We have $u_{2} v_{1}^{\prime} \in E$, for otherwise $u_{2} v_{1} \in F$ and $u_{1}^{\prime} v_{1}^{\prime} \in E_{b}$ would be in conflict in $G$ (recall that $F \subseteq E_{b} \cup E_{u}$ ). If $u_{2} v_{1}^{\prime} \in E_{r}$, then the vertices $u_{1}, v_{1}, u_{2}, v_{1}^{\prime}$ would induce a configuration $(C)$, a contradiction. Therefore, $u_{2} v_{1}^{\prime} \in E_{b} \cup E_{u}$. Similarly, since $u_{2} v_{2} \in E_{r}$, there is an edge $u_{2}^{\prime} v_{2}^{\prime} \in E_{b}$ such that $u_{2} v_{2}$ and $u_{2}^{\prime} v_{2}^{\prime}$ are in conflict in $G$, that is, $u_{2} v_{2}^{\prime}, u_{2}^{\prime} v_{2} \in$ $\hat{E}$. We have $u_{1} v_{2}^{\prime} \in E$, for otherwise $u_{1} v_{2} \in F$ and $u_{2}^{\prime} v_{2}^{\prime} \in E_{b}$ would be in conflict in $G$. Since $u_{1} v_{2}^{\prime}$ and $u_{2} v_{1}^{\prime} \in E_{b} \cup E_{u}$ are in conflict in $G$, we have $u_{1} v_{2}^{\prime} \in E_{r}$ and $u_{2} v_{1}^{\prime} \in E_{b}$. However, the vertices $u_{2}, v_{2}, u_{1}, v_{2}^{\prime}$ induce a configuration $(C)$, a contradiction. Thus, there are no alternating cycles of $E_{r}$ with length 4 relative to $G-F$.

We now suppose that there are an alternating cycle of $E_{r}$ with length grater than 4 relative to $G-F$. Let $A C$ be such an alternating cycle with minimal length, and let $u_{0}, v_{0}, u_{1}, v_{1}, \ldots u_{k-1}, v_{k-1}$ be the consecutive vertices of $A C$ with $u_{i} v_{i} \in \hat{E} \cup F$ and $u_{i+1} v_{i} \in E_{r}$ for any $i, 0 \leq i<k$ (indices are modulo $k$ ).

We claim that $A C$ has no edges in $F$. Suppose $u_{1} v_{1} \in F$. We have $u_{2} v_{0} \in E$, for otherwise the vertices $u_{2}, v_{1}, u_{1}, v_{0}$ would induce a configuration $(C)$. If $u_{2} v_{0} \in E_{r}$, then the vertices $u_{0}, v_{0}, u_{2}, v_{2}, \ldots u_{k-1}, v_{k-1}$ form a shorter alternating cycle of $E_{r}$ relative to $G-F$, contradicting the minimality of $A C$. Therefore, $u_{2} v_{0} \in E_{b} \cup E_{u}$. Since $u_{1} v_{0} \in E_{r}$, there is an edge $u_{1}^{\prime} v_{0}^{\prime} \in E_{b}$ such that $u_{1} v_{0}$ and $u_{1}^{\prime} v_{0}^{\prime}$ are in conflict in $G$, that is, $u_{1}^{\prime} v_{0}, u_{1} v_{0}^{\prime} \in$ $\hat{E}$. Similarly, since $u_{2} v_{1} \in E_{r}$, there is an edge $u_{2}^{\prime} v_{1}^{\prime} \in E_{b}$ such that $u_{2} v_{1}$ and $u_{2}^{\prime} v_{1}^{\prime}$ are in conflict in $G$, that is, $u_{2}^{\prime} v_{1}, u_{2} v_{1}^{\prime} \in \hat{E}$. The edges $u_{1}^{\prime} v_{0}^{\prime}$ and $u_{2}^{\prime} v_{1}^{\prime}$ are not the same edge, for otherwise $u_{1}^{\prime} v_{0}^{\prime} \in E_{b}$ and $u_{2} v_{0} \in E_{b} \cup E_{u}$ would be in conflict in $G$. Then, the vertices $u_{1}, v_{1}, u_{2}^{\prime}, v_{1}^{\prime}, u_{2}, v_{0}, u_{1}^{\prime}, v_{0}^{\prime}$ form an alternating cycle of $E_{b} \cup E_{u}$ relative to $G$ (recall that 
$F \subseteq E_{b} \cup E_{u}$ ). It follows from Lemma 5 that $E_{b} \cup E_{u}$ does not induce a chain graph, contradicting Lemma4 Thus, $A C$ has no edges in $F$.

Recall that the length of $A C$ is at least 6 , and let $u_{0}, v_{0}, u_{1}, v_{1}, u_{2}, v_{2}$ denote the consecutive vertices of $A C$. Since $A C$ has no edges in $F$, we have $u_{0} v_{0}, u_{1} v_{1}, u_{2} v_{2} \in \hat{E}$ and $u_{1} v_{0}, u_{2} v_{1} \in E_{r}$. We have $u_{2} v_{0} \in E$, for otherwise $u_{1} v_{0} \in E_{r}$ and $u_{2} v_{1} \in E_{r}$ would be in conflict in $G$. If $u_{2} v_{0} \in E_{r}$, then the vertices $u_{0}, v_{0}, u_{2}, v_{2}, \ldots u_{k-1}, v_{k-1}$ form a shorter alternating cycle of $E_{r}$ relative to $G-F$, contradicting the minimality of $A C$. Therefore, $u_{2} v_{0} \in E_{b} \cup E_{u}$. On the other hand, if $u_{0} v_{1} \in \hat{E}$, then the vertices $u_{0}, v_{1}, u_{2}, v_{2}, \ldots u_{k-1}, v_{k-1}$ form a shorter alternating cycle of $E_{r}$ relative to $G-F$, contradicting the minimality of $A C$. Therefore, $u_{0} v_{1} \in E$. Since $u_{0} v_{1}$ and $u_{1} v_{0} \in E_{r}$ are in conflict in $G$, we have $u_{0} v_{1} \in E_{b}$. By similar arguments, we have $u_{1} v_{2} \in E_{b}$. Then, we have $u_{0} v_{2} \in E$, for otherwise $u_{0} v_{1} \in E_{b}$ and $u_{1} v_{2} \in E_{b}$ would be in conflict in $G$. Since $u_{0} v_{2}$ and $u_{2} v_{0} \in E_{b} \cup E_{u}$ are in conflict in $G$, we have $u_{0} v_{2} \in E_{r}$ and $u_{2} v_{0} \in E_{b}$. This implies that the vertices $u_{1}, v_{0}, u_{2}, v_{1}$ induce a configurations $\left(B_{1}\right)$, a contradiction.

Thus, we conclude that there are no alternating cycles of $E_{r}$ relative to $G-F$.

Now, we have the following from Lemmas 5 and 6

Lemma 7. There is a chain completion of $E_{r}$ in $G-F$, and it can be computed in linear time from $E_{r}$.

Since every edge of $G$ belongs to either $E_{r}$ or $E_{b} \cup E_{u}, G$ can be covered by the chain completion of $E_{r}$ in $G-F$ and the chain subgraph of $G$ induced by $E_{b} \cup E_{u}$. Thus, we have the following from Lemmas 4 and 7

Lemma 8. From a given $(A, B, C)$-free bipartition of $E_{c}$, a 2-chain subgraph cover $\left(G_{1}, G_{2}\right)$ of $G$ such that $G_{1}$ has no edges in $F$ can be computed in linear time.

\section{Concluding Remarks}

This paper provides an $O\left(m \hat{m}+\min \left\{m^{2}, \hat{m}(\hat{m}+f)\right\}\right)$-time algorithm to solve the restricted 2-chain subgraph cover problem by reducing it to 2SAT. To do this, we show that the problem has a feasible solution if and only if there is an $(A, C)$-free bipartition of the set of committed edges of the given bipartite graph. This result implies a simpler recognition algorithm for simple-triangle graphs.

We finally note that for simple-triangle graphs, structure characterizations as well as the complexity of the graph isomorphism problem still remain open questions.

\section{Acknowledgments}

We are grateful to anonymous referees for careful reading and helpful comments. A part of this work was done while the author was in Tokyo Institute of Technology and supported by JSPS Grant-in-Aid for JSPS Fellows (26.8924). The final publication is available at Springer via http://dx.doi .org/10.1007/978-3-319-53925-6_14 


\section{References}

1. Aspvall, B., Plass, M.F., Tarjan, R.E.: A linear-time algorithm for testing the truth of certain quantified boolean formulas. Inf. Process. Lett. 8(3), 121-123 (1979)

2. Bogart, K.P., Laison, J.D., Ryan, S.P.: Triangle, parallelogram, and trapezoid orders. Order 27(2), 163-175 (2010)

3. Cerioli, M.R., de S. Oliveira, F., Szwarcfiter, J.L.: Linear-interval dimension and PI orders. Electron. Notes in Discrete Math. 30, 111-116 (2008)

4. Colbourn, C.J.: On testing isomorphism of permutation graphs. Networks 11(1), 13-21 (1981)

5. Corneil, D.G., Kamula, P.A.: Extensions of permutation and interval graphs. Congr. Numer. 58, 267-275 (1987)

6. Dagan, I., Golumbic, M.C., Pinter, R.Y.: Trapezoid graphs and their coloring. Discrete Appl. Math. 21(1), 35-46 (1988)

7. Dantas, S., de Figueiredo, C.M.H., Golumbic, M.C., Klein, S., Maffray, F.: The chain graph sandwich problem. Annals OR 188(1), 133-139 (2011)

8. Golumbic, M.C., Kaplan, H., Shamir, R.: Graph sandwich problems. J. Algorithms 19(3), 449-473 (1995)

9. Golumbic, M.C., Rotem, D., Urrutia, J.: Comparability graphs and intersection graphs. Discrete Math. 43(1), 37-46 (1983)

10. Hell, P., Huang, J.: Two remarks on circular arc graphs. Graphs Combin. 13(1), 65-72 (1997)

11. Ibaraki, T., Peled, U.: Sufficient conditions for graphs to have threshold number 2. Ann. Discrete Math. 11, 241-268 (1981)

12. Klavík, P., Kratochvíl, J., Krawczyk, T., Walczak, B.: Extending partial representations of function graphs and permutation graphs. In: Epstein, L., Ferragina, P. (eds.) ESA 2012. LNCS, vol. 7501, pp. 671-682. Springer Berlin Heidelberg (2012)

13. Lueker, G.S., Booth, K.S.: A linear time algorithm for deciding interval graph isomorphism. J. ACM 26(2), 183-195 (1979)

14. Ma, T.H., Spinrad, J.P.: On the 2-chain subgraph cover and related problems. J. Algorithms 17(2), 251-268 (1994)

15. Mahadev, N., Peled, U.: Threshold Graphs and Related Topics, Ann. Discrete Math., vol. 56. Elsevier Science B.V., Amsterdam, The Netherlands (1995)

16. McConnell, R.M., Spinrad, J.P.: Modular decomposition and transitive orientation. Discrete Math. 201(1-3), 189-241 (1999)

17. Mertzios, G.B.: The recognition of simple-triangle graphs and of linear-interval orders is polynomial. In: Bodlaender, H.L., Italiano, G.F. (eds.) ESA 2013. LNCS, vol. 8125, pp. 719-730. Springer Berlin Heidelberg (2013)

18. Mertzios, G.B.: The recognition of simple-triangle graphs and of linear-interval orders is polynomial. SIAM J. Discrete Math. 29(3), 1150-1185 (2015)

19. Raschle, T., Simon, K.: Recognition of graphs with threshold dimension two. In: Leighton, F.T., Borodin, A. (eds.) STOC 1995. pp. 650-661. ACM, New York, NY, USA (1995)

20. Shrestha, A.M.S., Tayu, S., Ueno, S.: On orthogonal ray graphs. Discrete Appl. Math. 158(15), 1650-1659 (2010)

21. Spinrad, J.P.: Circular-arc graphs with clique cover number two. J. Combin. Theory Ser. B 44(3), 300-306 (1988)

22. Spinrad, J.P.: Efficient Graph Representations, Fields Institute Monographs, vol. 19. American Mathematical Society, Providence, RI, USA (2003)

23. Takaoka, A.: Graph isomorphism completeness for trapezoid graphs. IEICE Trans. Fundamentals 98-A(8), 1838-1840 (2015) 
24. Takaoka, A., Tayu, S., Ueno, S.: Dominating sets and induced matchings in orthogonal ray graphs. IEICE Trans. Inf. \& Syst. 96-D(11), 2327-2332 (2014)

25. Uehara, R.: The graph isomorphism problem on geometric graphs. Discrete Math. \& Theoret. Comput. Sci. 16(2), 87-96 (2014)

26. Yannakakis, M.: The complexity of the partial order dimension problem. SIAM J. on Algebraic and Discrete Methods 3(3), 351-358 (1982)

\section{A Proof of Lemma 5}

Proof. We first prove the "only-if" part. A vertex of a bipartite graph is called an isolated vertex if it is not adjacent to any vertex, and a vertex is called a dominating vertex if it is adjacent to all the vertices on the other side of the bipartition. It is known that a chain graph has an isolated vertex or a dominating vertex [15]. Suppose $M$ has a chain completion $C$ in $H$, and there is an alternating cycle of $M$ relative to $H$. Since $C$ has all the edges in $M$, it also has all the vertices on the alternating cycle. Let $C^{\prime}$ be the subgraph of $C$ induced by the vertices on the alternating cycle. Since $C$ is a chain graph, $C^{\prime}$ is also a chain graph. However, $C^{\prime}$ has neither isolated vertex nor dominating vertex since any vertex of $C^{\prime}$ is incident to an edge in $M$ and incident to a non-edge in $\hat{E}$. It follows that $C^{\prime}$ is not a chain graph, a contradiction.

We next prove the "if" part by induction. We assume that the lemma holds for any bipartite graph with fewer vertices than $H$. Suppose there are no alternating cycles of $M$ relative to $H$. Then, $H$ has a vertex incident to no edges in $M$ or a vertex incident to no edges in $\hat{E}$, for otherwise we can grow a path alternating between $M$ and $\hat{E}$ until an alternating cycle is obtained. Let $u$ be such a vertex, and we assume without loss of generality that $u \in U$. Let $M-u$ be the set of edges in $M$ not incident to $u$, and let $H-u$ be the subgraph of $H$ obtained by removing $u$. Since there are no alternating cycles of $M$ relative to $H$, there are no alternating cycles of $M-u$ relative to $H-u$. It follows by induction that there is a chain completion $C=(U, V, E)$ of $M-u$ in $H-u$. If $u$ is incident to no edges in $M$, then $C^{\prime}=(U \cup\{u\}, V, E)$ is a chain completion of $M$ in $H$. If $u$ is incident to no edges in $\hat{E}$, then $C^{\prime}=(U \cup\{u\}, V, E \cup\{u v \mid v \in V\})$ is a chain completion of $M$ in $H$.

The proof of "if" part gives a linear-time algorithm that finds a chain completion of $M$ in $G$. The details of the algorithm are also shown in [7]. 